\title{
Valores evidenciales de o eso dicen
}

\author{
DOROTA KoTwICA \\ Investigadora Postdoctoral \\ Universitat de València, Grupo Val.Es.Co. \\ Facultat de Filologia, Traducció i Comunicació \\ Departamento de Filología Española \\ Av. Blasco Ibáñez 32, 46010 Valencia \\ E-mail: dorota.kotwica@uv.es
}

\section{VALORES EVIDENCIALES DE $O$ ESO DICEN}

RESUMEN: En este artículo se analizan los valores del significado evidencial transmitidos por la construcción o eso dicen. Se examinan los distintos patrones discursivos y sintácticos en los que dicha construcción aparece, así como su uso como marca de evidencialidad indirecta. El análisis se realiza a partir del Corpus de Español (sección Web/Dialects) y se centra en la variedad europea del español. Se concluye que o eso dicen transmite diferentes valores de evidencialidad, tales como significado reportativo, rumor y folclore que vienen determinados por factores relacionados con la fijación de la construcción, el tipo de la fuente de información a la que remite y el carácter del ámbito sobre el que opera. Asimismo, se comprueba que esta construcción a menudo sirve para mostrar el distanciamiento o desacuerdo del hablante con lo referido, aunque esta función parece diluirse en el caso de usos como marcador de folclore.

PALABRAS CLAVES: evidencialidad; reportativos; folclore; excepcionalidad reportativa; español.

SUMARIO: 1. Introducción. 2. La información procedente de otras personas dentro del dominio de la evidencialidad. 3. El análisis: patrones discursivos de o eso dicen y su relación con diferentes tipos de significado evidencial. 4. Conclusiones.

\section{EVIDENTIAL MEANINGS OF $O$ ESO DICEN}

ABSTRACT: In this paper, we analyze the evidential values transmitted by the Spanish construction o eso dicen ('or so they say'). We examine the different discursive and syntactic patterns in which this construction appears, as well as its use as a mark of indirect evidentiality. The analysis is carried out in the Corpus de Español (Web/Dialects section) and focuses on the European variety of Spanish. We conclude that o eso dicen transmits different values of evidentiality, such as report, rumor and folklore and this depends on factors related to the fixation of the construction, the type of the source of information to which it refers and the nature of its scope. Also, we confirm that that this construction often shows the speaker's distancing or disagreement with the reported information, although this function is not as prominent when it is used as a folklore marker.

KEY WORDS: evidentiality; reportatives; folklore; reportative exceptionality; Spanish.

SUMMARY: 1 . Introduction. 2 Information from other people within the domain of evidentiality. 3. The analysis: discursive patterns of $o$ eso dicen and its relationship with different types of evidential meaning. 4. Conclusions.

\section{VALEURS ÉVIDENTIELLES DE 0 ESO DICEN}

RÉSUMÉ: Dans cet article, nous analysons les valeurs évidentielles de la construction espagnole o eso dicen ('ou comme on dit'). Nous examinons les différents modèles discursifs et syntaxiques dans lesquels cette construction apparaît et son utilisation en tant que marque d'évidentialité indirecte. L'analyse est faite à partir du Corpus de Español (section Web/Dialects) et se concentre sur la variété européenne de l'espagnol. Nous concluons que o eso dicen transmet différentes valeurs d'évidentialité, telles que rapport, rumeur et folklore, qui sont déterminées par des facteurs liés à la fixation de la construction, au type de source d'information et à la nature de la portée. En outre, on constate que cette construction sert souvent à montrer la distance ou le désaccord du locuteur avec l'information référée, bien que cette fonction semble être moins importante dans le cas d'utilisations en tant que marqueur de folklore.

MOTS CLÉS: évidentialité; reportativité; folklore; reportative exceptionnalité; espagnol.

SOMMAIRE : 1 . Introduction. 2 L'information originaire d'autres personnes dans le domaine de l'évidentialité. 3. L'analyse : patrons discursifs de o eso dicen et son rapport avec différents genres de signification évidentielle. 4. Conclusions.
Fecha de Recepción Fecha de Revisión Fecha de Aceptación Fecha de Publicación
$30 / 11 / 2018$

$01 / 07 / 2019$

$30 / 07 / 2019$

$01 / 12 / 2019$ 


\section{Valores evidenciales de o eso dicen ${ }^{1}$}

\section{DOROTA KOTWICA}

\section{INTRODUCCIÓN}

En español existen diversas estrategias lingüisticas para señalar que alguna parte de la información procede de fuentes ajenas al propio hablante, esto es, que se trata de valores reportativos o citativos de la evidencialidad indirecta. De esta manera funciona la construcción o eso dicen en el siguiente ejemplo:

(1) "Hoy en día, la casa ha sido declarada Historical Landmark por el gobierno de California. Algunos todavía creen que en ella moran unos cuantos fantasmas. Expertos en lo paranormal que han acudido a la casa han salido, o eso dicen, convencidos de que es así. Los hay que han visto luces extrañas o los que han oído ruidos sospechosos (CDE/WebDialects, cabovolo.com)". ${ }^{2}$

En el ejemplo (1), o eso dicen marca que la fuente de la afirmación "han salido convencidos de que es asi" no es el propio hablante, sino otras personas. Más concretamente, se alude a las palabras de los "expertos en lo paranormal".

La asociación de o eso dicen con la información que proviene de fuera del hablante (del discurso de otras personas) es tan fuerte que, de hecho, esta construcción ha sido utilizada para corroborar el carácter citativo de otros elementos evidenciales. Encontramos un ejemplo de ello en el trabajo de Cornillie (2016: 239-240), quien utiliza o eso dicen a modo de apéndice en las oraciones con verbos auxiliares evidenciales para comprobar si tienen carácter citativo y, además, si existe la disociación entre la conceptualización del hablante y la información reportada. Como argumenta Cornillie (2016), cuando la añadidura de o eso dicen a una oración marcada con un elemento evidencial no resulta problemática, estamos ante un caso de evidencialidad reportativa en la que esta disociación es posible.

En el caso de o eso dicen el valor de disociación de la perspectiva del hablante con la información reportada parece venir asegurado por la presencia del elemento disyuntivo en la construcción (la conjunción o) que por sí solo sugiere que la información señalada deícticamente por medio de eso resulta poco fiable, incierta o, simplemente, no verdadera a ojos del hablante. Parece, por tanto, que o eso dicen es un elemento predilecto para introducir lo dicho por otros y, además, para poner la veracidad de esta afirmación ajena en duda o negarla.

\footnotetext{
${ }^{1}$ Esta investigación se ha realizado en el marco del proyecto postdoctoral APOSTD/2019/161 financiado por la Generalitat Valenciana y el Fondo Social Europeo.

${ }^{2}$ Los ejemplos del corpus se citan literalmente.
} 
Sin embargo, cabe replantear las afirmaciones anteriores ante los ejemplos como el (2). Y es que resulta que, aparte de los usos que intuitivamente podrian considerarse prototípicos -en los que el hablante se distancia de lo reportado, mostrándose escéptico o incrédulo- o eso dicen a menudo marca también oraciones en las que se citan frases de sabiduría popular, dichos y refranes, como en (2), en los que el distanciamiento del hablante parece reducirse o desaparecer.

(2) "Buenos dias! Superado el lunes superada toda la semana, o eso dicen, ¿no?” (CDE/WebDialects, mypeeptoes.com)

La peculiaridad de los ejemplos como (2) consiste en que, al citar una frase procedente del acervo común ("superado el lunes superada toda la semana"), a pesar de emplear o eso dicen, el hablante no parece contradecir el contenido citado o desligarse por completo del mismo. Por el contrario, parece que se alinea con lo dicho y utiliza la frase hecha para reforzar su propia opinión o punto de vista. Busca, asimismo, solidaridad con el lector, no solo por medio de citar una frase conocida por ambos, sino también por el uso del marcador ¿no?

¿Qué es lo que diferencia los ejemplos (1) y (2) a nivel del significado evidencial? En ambos casos, ¿se trata de usos reportativos? Y si es así, ¿dónde reside la peculiaridad de los contextos como (2) en los que el hablante no parece poner en entredicho lo que dicen los demás?

Nuestro cometido en el presente trabajo es tratar de contestar a las anteriores preguntas. Para ello, observaremos en qué patrones discursivos aparece o eso dicen y cómo estos patrones se relacionan con el tipo de significado evidencial que transmite.

\section{LA INFORMACIÓN PROCEDENTE DE OTRAS PERSONAS DENTRO DEL DOMINIO DE LA EVIDENCIALIDAD}

El español se reconoce como lengua no evidencial, en el sentido de que la expresión de la categoría de la evidencialidad no es obligatoria ni se realiza por una clase específica de elementos gramaticales (véase, por ejemplo, Albelda, 2015). Sin embargo, resulta evidente que el español cuenta con diversas maneras de expresar los significados evidenciales, esto es, aquellos relacionados con la procedencia de la información. En esta ocasión, nos interesan, especialmente, aquellos elementos lingüísticos que se dedican a introducir la información procedente de otras personas. Como apunta Aikhenvald (2004: 132): "Every language has some ways of reporting what someone else said. The speaker can use their own words, or quote the other person verbatim". La citada autora señala las similitudes entre estas estrategias y los evidenciales "legítimos" reportativos o citativos. Diferentes construcciones que introducen discurso directo o indirecto a menudo pueden 
verse como "paráfrasis léxicas" de aquellos elementos que se encuentran gramaticalizados en los sistemas evidenciales (Aikhenvald, 2007: 218).

En cuanto al propio significado evidencial reportativo o citativo, este forma parte el dominio de la evidencialidad indirecta, dentro del cual, en muchas clasificaciones, se incluyen también las inferencias y las evidencias procedentes del razonamiento de las que no nos ocuparemos en esta ocasión. En la conocida clasificación de Willet (1988: 57), la distinción entre directo e indirecto se relaciona con el carácter primario o secundario de la fuente de información. Entre los tipos indirectos de evidencia se encuentran los reportativos que pueden proceder de segunda mano, tercera mano o folclore, relacionado este último con la "literatura oral", además de las inferencias.

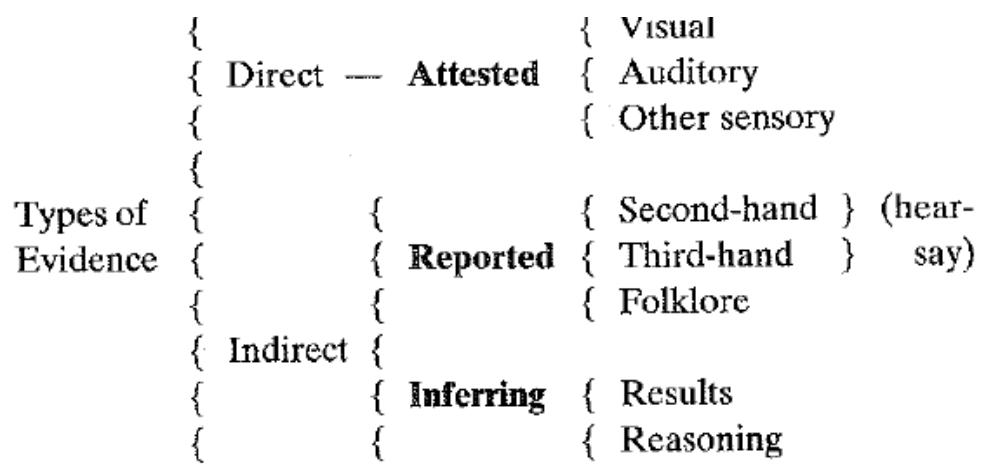

Figure 1: Types of evidence

Figura 1: Clasificación de evidencialidad según Willet (1988)

Como explica Plungian (2001), los citativos ("quotative") son el único tipo de evidencialidad realmente mediada. Su característica más destacada y la que la diferencia de otros tipos evidenciales es que proceden de otras personas, por lo cual el hablante, normalmente, no tiene ninguna relación directa con la información marcada mediante un elemento evidencial, como se explica en la siguiente cita:

However, quotative is the only evidential value which refers to a true mediated knowledge (in the sense of Lazard, 1956, Guentcheva, 1990). In all the other cases, the speaker either has access to $\mathrm{P}$ as such or, at least, to some indirect manifestations of $\mathrm{P}$. Quotative signals that the speaker is separated from the situation by the barrier of another observer (...). Thus, the possibility of being the most indirect evidence about $\mathrm{P}$ is reserved for quotative. In this respect, one can speak about 'speaker's personal involvement': nonquotative indirect uses presuppose that the speaker is still associated with $P$ (when observing, for example, its traces), though s/he does not perceive P directly. In contrast, quotative uses normally exclude any personal involvement. (Plungian, 2001: 353)

Cabe advertir de que la evidencialidad procedente de otras personas a veces recibe la denominación reportativa/reportada/referida $\mathrm{y}$, otras veces, 
citativa. Si bien, en algunos casos, podría tratarse simplemente de una confusión terminológica, en otros, la distinción entre lo reportado y lo citativo queda perfectamente justificada por factores relativos a la concreción de la fuente de información a la que se hace referencia. En este sentido, parece especialmente interesante la subdivisión de las evidencias indirectas procedentes del discurso de otros que se hace con relación a la presencia o no de la fuente indirecta responsable de la información, de la que habla Aikhenvald (2007):

Reported, for reported information with no reference to who it was reported by (...) Quotative, for reported information with an overt reference to the quoted source. (Aikhenvald, 2007: 211)

Por su parte, Lampert y Lampert (2010: 311) hacen una distinción triple entre a) reportativos (reportative) que señalan el modo de obtener el conocimiento, pero no necesariamente la fuente (por ejemplo, they say, it is said, $s /$ he is said); b) citativos (quotative) que destacan la fuente, pero no siempre señalan el modo de obtener la información (por ejemplo, according to somebody/something, y c) reportativo-citativos (reportative-quotative) en los que se especifica tanto el modo de obtener el conocimiento como la fuente (por ejemplo, los verbos de cita).

La presencia explicita (o no) de la fuente ajena responsable de una aserción resulta relevante, por ejemplo, en el discurso científico, en el cual se materializa por medio del sistema de referencias bibliográficas. Como señala Chafe (1986: 268-269), las citas y las referencias son recursos formales ("formal devices") para expresar el conocimiento obtenido por medio del lenguaje $^{3}$ en el discurso académico. Existen, además, propuestas de clasificaciones mucho más pormenorizadas que también tienen en cuenta la presencia explícita de la fuente y su formato. Por ejemplo, la clasificación de Dehkordi y Allami (2012) se organiza en torno al grado de especificidad en los mecanismos de referencia y citación con lo cual se consigue una caracterización muy completa de las maneras en las que las evidencias reportadas aparecen en el discurso científico. Además de las citas directas y referencias, tienen cabida también evidencias no específicas ambiguas que enfatizan los hechos más que la fuente, y esta última no se menciona, por ejemplo, It is generally believed that... (Dehkordi y Allami, 2012: 1901).

La información procedente de la sabiduría popular o conocimientos compartidos también a menudo se relaciona con el dominio de la reportatividad $\mathrm{o}$, al menos, de la evidencia indirecta procedente de las fuentes externas. En las clasificaciones de Bermúdez (2005), la de Plungian (2001) o la de Willet (1988), folclore se encuentra dentro del dominio de evidencialidad indirecta procedente de otros.

\footnotetext{
${ }^{3}$ En su clasificación de significados evidenciales, Chafe (1986: 262) subraya que una de las fuentes de conocimiento es el lenguaje, de esta proceden evidencias denominadas hearsay.
} 
Bermúdez (2005) postula que el dominio de la evidencialidad debe organizarse en torno a tres ejes: el modo de adquisición de la información, o cómo se ha accedido a la información (de manera cognitiva o sensorial), la fuente de información, o dónde se ha obtenido la información (personal o ajena) y el acceso a la información o quiénes comparten la fuente. En esta clasificación, las evidencias reportativas se distinguen por proceder de una fuente ajena y no accesible ("Y por defecto el acceso es privativo, dado que el hablante informa al oyente sobre la información que recibió de fuente ajena", citando a Bermúdez (2005: 17), mientras que el folclore, que también forma parte del dominio de evidencialidad indirecta que procede de otros "se sitúa en un tipo de conocimiento que es común y de acceso garantizado para todos los integrantes de una comunidad, tipicamente transmitido por vía oral. De esta manera se explica la diferencia sustancial entre ambas nociones [reportativo y folclore]" (Bermúdez, 2005: 17).

Por su parte, Lazard (2001), en un comentario sobre la propuesta de Plungian (2001), sugiere que debería guardarse un espacio propio dedicado a "los conocimientos procedentes de la tradición y conocimientos compartidos", pues postula que su naturaleza es distinta de la de los evidenciales de segunda mano o hearsay. Cabe señalar, sin embargo, que solo en escasos sistemas evidenciales gramaticales existen marcadores específicos de conocimientos compartidos (Plungian, 2010: 32). Es así, por ejemplo, en el idioma calmuco, en cuyo sistema evidencial se distinguen los siguientes siete valores de evidencialidad: directa, indirecta, inferida, asumida, prospectiva, reportada y de conocimientos compartidos (Aikhenvald, 2018). También en el idioma buriato existen marcas gramaticalizadas de evidencialidad para la reportatividad y los conocimientos compartidos.

Como se ha podido ver hasta aquí, en las diferentes clasificaciones de los evidenciales siempre tienen cabida las evidencias procedentes de otras personas, aunque pueden recibir denominaciones diferentes y pueden subdividirse en más categorias específicas, si se tiene en cuenta la especificidad de la fuente o el tipo de acceso.

Además de apuntar a las fuentes de conocimiento externas al hablante, los recursos de evidencialidad reportativa comparten la peculiaridad conocida como "excepcionalidad reportativa" (reportative exceptionality) (AnderBois, 2014). Se ha demostrado que los reportativos son el único tipo evidencial que puede introducir evidencia para información con la que el hablante no se alinea del todo o con la que no está de acuerdo. Esto es posible porque, como explica AnderBois (2014), con los reportativos ocurre un cambio de perspectiva (perspective shift) del que hablaron Harris y Pott (2009). Asimismo, la proposición marcada evidencialmente por un reportativo no es asertada (a diferencia de otros tipos de evidencialidad), sino que es presentada por el hablante (Faller, 2002: 135). Esto tiene ciertas consecuencias a nivel del compromiso del hablante con lo dicho: "The speaker publicly commits herself to having a certain type of evidence for $p$, but avoids having to make a public commitment to $p$ itself" (AnderBois, 2014: 20), 1o 
cual con los reportativos es incluso más fácil que con otros evidenciales, gracias a su excepcional naturaleza que permite el cambio de perspectiva ("the proposal is attributed to the reporter rather than the speaker herself", AnderBois, 2014: 32).

Esta excepcionalidad de los reportativos nos parece especialmente relevante a la hora de analizar el caso de o eso dicen que, que, a primera vista, parece ser un perfecto elemento reportativo "excepcional" cuya función sería la de señalar que la opinión del hablante es distinta a la que se expresa en la proposición reportada.

En cuanto a la expresión de los significados evidenciales reportativos en español, existen, sin duda, diversas "estrategias o expresiones evidenciales" que se ocupan de su trasmisión (González Vázquez, 2016: 57). Podríamos mencionar, por ejemplo, los verbos de lengua (dicen que, se dice que, según dicen según González Vázquez (2016: 58)), además de algunos usos de tiempos y modos verbales (el imperfecto del indicativo, Reyes 1994, Hassler 2010; el futuro, Escandell Vidal 2010, Rodriguez Rosique 2015, García Negroni 2016, entre otros), que citativo (Gras 2016, entre otros), etc.

En algunas variedades americanas, el español cuenta también con ciertos marcadores de evidencialidad indirecta convencionalizados como dizque (Miglio 2010, la Mora y Maldonado 2015). Dizque se ha considerado tradicionalmente como un marcador de evidencialidad reportativa (Miglio 2004), además, en sus usos formulaicos (aquellos que parecen impersonales, no definen en ningún momento quién es el que dice que), dizque apunta también a conocimientos comunes o proverbios (Miglio, 2010: 17). Además de expresar el significado evidencial, dizque se asocia con el significado epistemico: "the epistemic meaning of dizque, or the meaning whereby the speaker expresses mistrust in the information s/he reports" (Miglio, 2010: 19). Para Miglio (2010) se trata de un significado secundario y derivado del significado evidencial. Por su parte, De la Mora y Maldonado (2015: 171) señalan que, en sus usos actuales en el español mexicano, el significado reportativo se está extinguiendo a favor de un significado epistémico:

we show that epistemic meanings have become the core of dizque and that the reportative meaning is dying out in Mexican Spanish. Instead, two basic components coexist: a weakened evidential-reportative and an epistemic meaning questioning the veracity of events and things being referred to. We show that the source of information has become less relevant than the truthfulness of the information being conferred. (De la Mora y Maldonado, 2015: 171)

El caso de dizque parece especialmente interesante en relación con la construcción que analizamos en este trabajo, o eso dicen, que también se asocia, especialmente, con usos en los que la veracidad de la información reportada está cuestionada y, además, como hemos visto en el ejemplo (2), puede acompañar proverbios y dichos de la sabiduría popular. 


\section{EL ANÁlISIS: PATRONES DISCURSIVOS DE O ESO DICEN Y SU RELACIÓN CON DI- FERENTES TIPOS DE SIGNIFICADO EVIDENCIAL}

Se han analizado las ocurrencias de o eso dicen en el subcorpus Web/Dialects del Corpus de Español de Mark Davies, seleccionando únicamente la variedad europea del español (https://www.corpusdelespanol.org/). Los parámetros del análisis han sido los siguientes: modo de inserción (parentético/no parentético), presencia del sujeto u otros complementos de decir y presencia de marcas adicionales del rechazo por parte del hablante hacia la información reportada. Además, se ha reflexionado acerca del significado evidencial que introduce esta construcción en diferentes contextos, partiendo de que el significado básico que se presupone es el reportativo, tal como sugiere su significado composicional y la presencia del verbo decir.

\subsection{LOS PATRONES DE INSERCIÓN DE O ESO DICEN EN EL DISCURSO}

En total, se han analizado 195 ocurrencias de o eso dicen y se han delimitado tres patrones en los que aparece en el discurso, en cuanto a su estructura e inserción. En primer lugar, se han visto usos de o eso dicen integrado en la estructura de la oración, con un sujeto adyacente, como en (3) o algún complemento del predicado que acompaña a toda la estructura, como en el ejemplo (4). Se trataria, por tanto, de usos plenamente composicionales de esta construcción.

(3) "El todo no tiene ni principio ni fin (ni propósito) o eso dicen los dos mayores cerebros del momento especialistas en ello: Hawkings y Penrouse" (CDE/WebDialects, recuerdosdepandora.com)

(4) "Actualmente existen algunas agrupaciones que realmente se preocupan por la gente, o eso dicen desde la parte baja de los de arriba. Yo quiero cambiar muchas cosas" (CDE/WebDialects, bordes.org)

Estos usos no parentéticos suponen una minoría dentro del todo el conjunto de ocurrencias analizados (22\%).

En segundo lugar, distinguimos los usos parentéticos, en los que o eso dicen aparece a modo de inciso (separado por medio de comas, paréntesis u otras marcas tipográficas), aunque la forma dicen concuerda con algún elemento de la oración o del contexto más amplio. Es decir, se puede recuperar con bastante facilidad quiénes son las personas a las que se les atribuye 1o dicho. Este sería el caso del ejemplo citado en (5), donde o eso dicen concuerda con "los políticos":

(5) "Pero cuando creo que puedo, adquiero la capacidad de hacer lo aun si antes no la tenía Pues yo no lo veo..... los políticos creen (o eso dicen) 
que están al servicio de la sociedad y, en realidad, están al servicio de sus bolsillos y grandes multinacionales y organizaciones religiosas ¿falla el refrán? ¿o es que además de todo, los políticos mienten?” (CDE/WebDialects, frasesparalahistoria.com)

En ejemplos como el anterior (5), o eso dicen conserva su significado composicional, al referirse a un sujeto en tercera persona del plural que aparece en alguna parte del contexto. Sin embargo, ocurren también casos como (6) en los que, si bien podemos determinar a qué grupo de personas remite el verbo decir, estas no aparecen directamente citadas en el contexto.

(6) "Comenzando por la primera afirmación, el objetivo de Mark Shuttleworth de hacer del escritorio Linux algo más atractivo que Mac OS X a la vista, se ha cumplido, según publican nuestros compañeros de MuyComputer. Por lo menos desde el punto de vista del sudafricano (jo eso dicen en la fuente de la noticia!). No deja de ser una apreciación personal, que puede generar algo de discusión, pero que no va más allá" (CDE/WebDialects, muylinux.com)

Así, en el fragmento (6), se menciona de dónde proviene la afirmación citada ("en la fuente de la noticia"), aunque no se señala con más precisión quiénes exactamente son los responsables de lo dicho.

El tercer patrón son los usos parentéticos impersonales, como en (7). En este tipo de contextos, toda la construcción de o eso dicen adquiere un carácter impersonal, pues no remite a palabras de nadie específico, sino que señala que se trata de lo que dicen algunos o todos, personas no específicas.

(7) "Ella no entiende cómo puedo estarme parada de pie derecho sin moverme durante mucho tiempo. Tendría que hacer yoga para aprender estas cosas. O eso dicen" (CDE/WebDialects)

La frecuencia de aparición de cada uno de estos tres patrones en los que o eso dicen se inserta en el discurso se ilustra en la Figura (2).

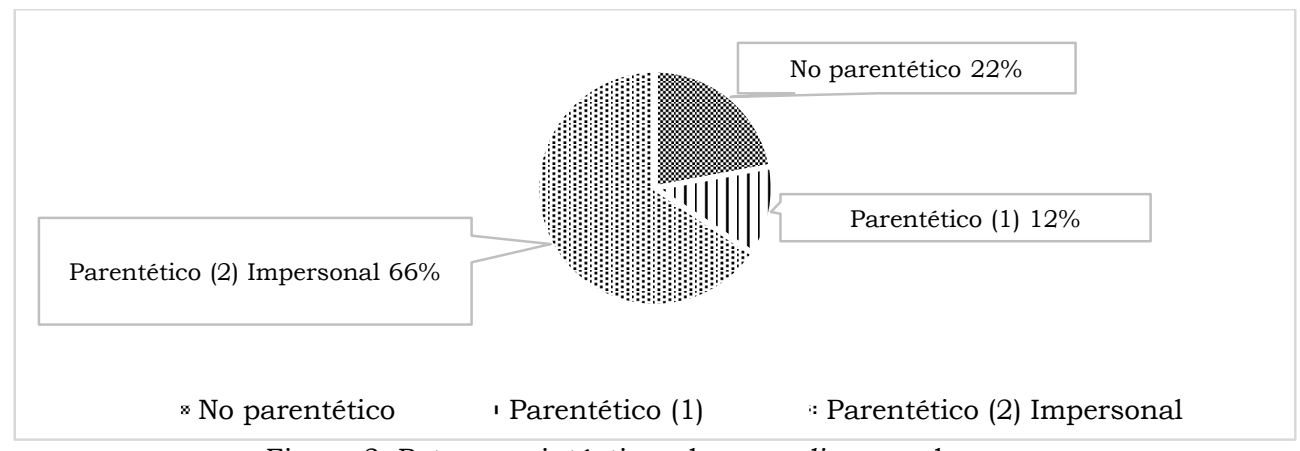

Figura 2: Patrones sintácticos de o eso dicen en el corpus 
Como se puede observar, predominan los usos parentéticos de o eso dicen de carácter impersonal (66 \%). En segundo lugar, en $22 \%$ de los casos, esta construcción aparece como integrada en el discurso, con una presencia explícita del sujeto o de algún complemento del verbo. Finalmente, en un 12 $\%$ de los contextos, o eso dicen formalmente aparece como parentético, aunque, en realidad, existe un referente de la forma dicen en alguna parte del contexto próximo 4 .

Estos resultados demuestran que no se trata de una construcción completamente fijada ${ }^{5}$, asi que no podemos hablar de un recurso evidencial plenamente convencionalizado, como podría ser el americano dizque. Sin embargo, dado que nuestra perspectiva a la hora de definir la evidencialidad en español es amplia, no rechazamos los usos de las estrategias lingüísticas como los casos de o eso dicen con un significado composicional, pues no son muy diferentes de otros usos de verbos de comunicación que informan sobre la existencia del acto de decir algo y, secundariamente, pueden relacionarse con la expresión de la evidencialidad reportativa.

Consideramos que el hecho de que coexistan los patrones más o menos fijados de esta construcción resulta especialmente interesante desde el punto de vista del valor evidencial que se transmite. Se podría hablar de una escala de explicitud en cuanto a la expresión de la fuente de información, desde una presencia patente (o eso dicen los expertos), pasando por una presencia contextual (los expertos han encontrado una solución..., o eso dicen), hasta una total ausencia de la fuente o la impersonalidad. Como trataremos de demostrar a continuación, cada uno de estos patrones está asociado a unos matices diferentes del significado evidencial.

\subsection{EL SIGNIFICADO EVIDENCIAL}

Como hemos señalado anteriormente, o eso dicen aparece a menudo con el sujeto, que puede estar adyacente a la construcción o recuperable del contexto. Por tanto, además de señalar que cierta parte de la información proviene del discurso ajeno, se aporta alguna información acerca de la fuente indirecta a la que remite el hablante. Se podria pensar que en todos estos casos se trata de la llamada evidencialidad citativa, pues al mencionar el sujeto responsable de la afirmación, queda expuesta la fuente. Sin embargo, el grado de especificación de la fuente es tan variable que, aunque sintácticamente el sujeto esté presente, a menudo apenas da pistas acerca del responsable de una aserción. Veamos algunos ejemplos.

\footnotetext{
${ }^{4}$ No es nuestro cometido el análisis sintáctico de esta construcción, aunque sí opinamos que la presencia de estos tres patrones resulta interesante también desde el punto de vista del análisis del significado evidencial asociado con o eso dicen.

${ }^{5}$ Del corpus se recuperan otras formas en las que ocurren cambios en el tiempo verbal, en la persona, aparecen elementos intercalados, etc., por ejemplo, o eso se decía, o eso me dijeron.
} 
En el ejemplo (8), se está hablando de las acciones prometidas por una entidad concreta, el grupo Bankia. Podemos sospechar, asimismo, que se trata de los representantes del mismo grupo quienes han hecho pública la información a la que se alude ("al final de este mes, va a canjear esas preferentes"), aunque no podemos llegar a concretar una fuente más específica.

(8) "A finales de este mes, o eso dicen, el grupo Bankia, va a CANJEAR estas preferentes...pero AÚN NO SE SABE SI SERÁ EN ACCIONES, EN BONOS..." (CDE/WebDialects, burbuja.info)

Por su parte, en (9) la única información que se da acerca de la fuente es que son "los hombres de la ciencia" lo cual resulta sumamente impreciso.

(9) "No entro en el debate en sí de si fiesta sí o fiesta no, al final con el tiempo desaparecerá y tendremos que adaptar nos, sin más, así es como se evoluciona o eso dicen los hombres de ciencia ;-)..” (CDE/WebDialects, cienciaxxi.com)

Si la fuente en los ejemplos como los anteriores (8) y (9) queda difusa, se convierte en extremadamente vaga en casos como el siguiente (10), donde la aportación del sujeto que concuerda con decir ("algunos") resulta muy poco informativa y, en realidad, no permite de ninguna manera identificar a la fuente de la aserción.

(10) "De manera que no todo en la vida queda encajado en su propia ecuación existencial, porque la emoción es poder, lo mismo que el amor es la energía más poderosa que existe. O eso dicen algunos" (CDE/WebDialects, felicidadenlavida.com)

¿Hasta qué punto se puede hablar de la evidencialidad citativa en los casos como los anteriores? La presencia del verbo conjugado en la tercera persona del plural facilita que la construcción se utilice para evitar la especificación de la fuente, esto es, con una pretensión claramente opuesta a la que tendría una construcción citativa. Todos los ejemplos que encontramos en el corpus en los que aparece el sujeto comparten esta característica de aludir a un grupo muy difuso de personas responsables de la información (o eso dicen los psicólogos y los filósofos; o eso dicen los defensores; o eso dicen las estadísticas; o eso dicen los hombres de la ciencia; o eso dicen los testigos; o eso dicen los portugueses; o eso dicen algunos medios de comunicación, etc.), valga como una excepción rara el ejemplo (3), en el que se ponen los apellidos a "los que dicen".

En definitiva, dificilmente podemos clasificar como citativos este tipo de usos de o eso dicen como, ya que la fuente colectiva a la que remiten resulta demasiado vaga y general para que se le pueda considerar como una voz responsable de emitir una aserción. Sin embargo, la presencia del sujeto o 
de los complementos del decir conllevan una lectura literal de o eso dicen, sugieren un significado composicional y, efectivamente, parecen indicar que existen ciertas personas que dicen algo o que han dicho algo; se remite a un acto de habla más concreto y específico. Parece, pues, que se trata simplemente de una estrategia reportativa, en la que la intención del hablante es la opuesta que se esperaría de un elemento citativo, esto es, hacer que la fuente parezca lo más opaca posible.

Veamos ahora los usos parentéticos de o eso dicen impersonales. En cuanto al valor evidencial, creemos que, en este patrón discursivo, o eso dicen se desdobla en dos tipos de significados. Compararemos el ejemplo (11) frente al ejemplo (12) para ilustrarlo.

(11) "Hoy 5 equipos desean que gane el Rayo si o si, espero que no les demos ese gusto, además que casualidad que el entrenador del Rayo será el nuevo entrenador del Zaragoza (o eso dicen)... mas ganas tendrá de ganar entonces." (CDE/WebDialects, futboldesdemallorca.com)

(12) "Ya se sabe que la belleza hay que buscar la en el interior, o eso dicen. Como no podía ser de otro modo, su directo es brutal." (CDE/WebDialects, actualidad24.net)

Los dos ejemplos de arriba (11 y 12) presentan una estructura similar: $o$ eso dicen aparece de modo parentético y en ninguna parte del contexto se ofrece información acerca de la fuente responsable. Por su parte, las diferencias entre estos ejemplos residen en el tipo del ámbito al que afecta la construcción y en el carácter de la fuente. Al hablar del ámbito, nos referimos al tipo de información que viene marcada por o eso dicen, es decir, el segmento al que remite el deíctico eso dentro de la construcción. Así, el ámbito de o eso dicen en (11) sería "el entrenador del Rayo será el nuevo entrenador del Zaragoza". Se trata de una afirmación acerca de una situación puntual, no tiene carácter atemporal ni universal. Por su parte, en (12), la afirmación afectada, "la belleza hay que buscar la en el interior", es un dicho popular, una frase fijada en la tradición oral y con una validez que se presupone universal y atemporal.

En cuanto al carácter de la fuente, en (11) la autoría de la afirmación podría adjudicarse a un determinado grupo de personas (algún medio de comunicación, las noticias, etc.). Por su parte, en (12), la fuente son los hablantes que forman parte de una comunidad lingüistica y comparten una tradición oral.

Se ve, pues, que la diferencia entre ambos ejemplos radica en el grado de la universalidad, tanto de la afirmación marcada mediante o eso dicen, como de la fuente a la que esta construcción remite. En resumen, mientras que en el primer tipo (11) aún se percibe, aunque muy vagamente, cierta parte del significado composicional primario de esta construcción (se señala que alguien dice algo), esto no ocurre en (12), donde el valor del verbo decir se 
difumina casi por completo. En casos como el (12), ya no se trata de reproducir o parafrasear algo que otros han dicho, sino que se repiten afirmaciones consideradas como sabiduría general o conocimientos compartidos por parte de la comunidad a la que pertenece el hablante. También resulta interesante que el hablante apenas cuenta con un pequeño margen de creatividad a la hora de transmitir lo dicho, puesto que, al tratarse de frases fijadas en la tradición oral, tienen que citarse sin mayores alteraciones para que resulten inteligibles.

En definitiva, se observa que, cuando el uso de esta construcción se aleja de su significado composicional, pierde la relación con el significado básico de "algunas personas dicen algo" y adquiere un valor no solo impersonal, sino que también universal. Al mismo tiempo, también aumenta el grado de la abstracción y de la generalización en la información que se encuentra dentro del ámbito de o eso dicen. Observamos un movimiento desde un acto individual, particularizado de "decir algo alguien", hacia frases pertenecientes a la voz general del pueblo, atemporales y universales. Asimismo, si bien en los casos como (11), la parte del significado evidencial que se destaca es que la información procede de otros (es decir, la reportatividad propiamente dicha), en los contextos como (12), lo que se acentúa es la a dimensión del acceso, el carácter compartido y universal de la fuente. Podriamos decir, por tanto, que estos segundos contextos se relacionan más con la evidencialidad de tipo folclore.

A partir de lo dicho en el apartado anterior, creemos que para describir el funcionamiento evidencial de o eso dicen es necesario hablar de una escala o una gradación del significado. En primer lugar, existen los usos más reportativos, relacionados con la presencia explícita de la fuente, en los que el significado evidencial viene asegurado por el significado composicional de la construcción $o+$ eso + dicen. En segundo lugar, un caso intermedio serían los contextos en los que el significado composicional se va perdiendo, o eso dicen aparece como parentético y la fuente no se aprecia en el contexto, pero sigue delimitada a un grupo de personas; el hablante muestra la información casi como si fuera un rumor. Finalmente, cuando o eso dicen pierde la relación con el significado composicional, se desvanece el significado reportativo a favor del significado de folclore.

En cuanto a la presencia de estos diferentes valores del significado evidencial en el corpus analizado, aparecen en proporciones diferentes (véase la Figura 3). La mayor parte de las ocurrencias (47\%) se corresponde con los usos reportativos con matiz de rumor (es decir, el punto intermedio de la escala). Seguidamente, un $34 \%$ son los usos reportativos resultantes del significado composicional de la construcción. Finalmente, en un 19 \%, observamos los contextos que sugieren el valor de conocimientos compartidos o folclore. 


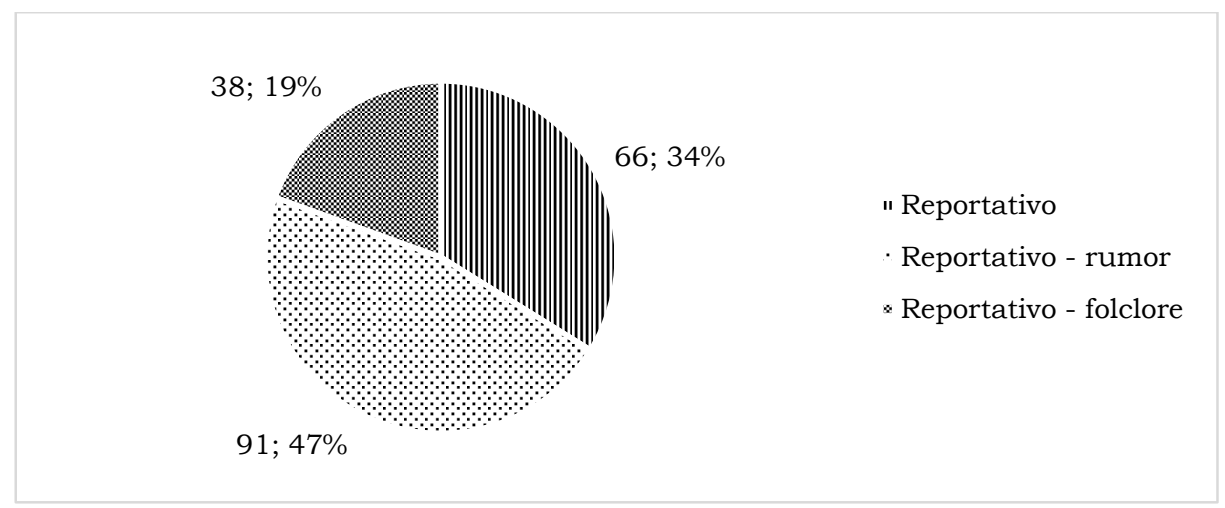

Figura 3: Valores del significado evidencial de o eso dicen en el corpus

Como se ha visto, los patrones sintácticos y discursivos en los que aparece o eso dicen son de gran ayuda a la hora de esclarecer su significado evidencial. Definitivamente, parece que, a mayor fijación de la construcción, menos precisa es la fuente y menos pronunciado es el significado reportativo.

\subsection{O ESO DICEN Y LA EXCEPCIONALIDAD REPORTATIVA}

Queda pendiente retomar la idea de que o eso dicen parece ser un marcador "predilecto" de la disociación, como sugeririan la prueba de Cornillie (2016) y la proximidad entre o eso dicen y dizque. A continuación, pues, trataremos de esclarecer cuál es la relación de o eso dicen con la excepcionalidad tan característica para los reportativos, de la que hablamos anteriormente en línea de las ideas de AnderBois (2014) y Faller (2002).

A primera vista, podría parecer que o eso dicen sugiere dicha disociación en todos los casos de su uso. La adición de "o eso" a esta construcción hace que su lectura sea diferente respecto a la lectura que tendria un uso simple del verbo de comunicación. Ilustrémoslo con unos ejemplos creados a propósito de esta discusión:

(13) "Tomar poca sal es lo mejor para la salud, dicen los especialistas."

(14) "Tomar poca sal es lo mejor para la salud, o eso dicen los especialistas."

Observemos cómo cambia la interpretación entre los ejemplos (13) y (14). En ambos, la información reportada es presentada en primer lugar y la marca evidencial viene a continuación. La fuente en ambos casos es la misma, poco definida y plural, "los especialistas". La principal diferencia es que en (13) nada indica que el hablante no se alinee con la opinión citada; es más, nada impide que se utilice aquí la cita de los expertos para aumentar la fiabilidad de la información, pues el hablante por sí solo podría no tener la autoridad suficiente como para afirmar que no se debe tomar mucha sal. 
En todo caso, la excepcionalidad reportativa se podría activar aquí sin ningún problema, aunque para ello sería necesario que el hablante añadiera algún elemento más a su enunciado, por ejemplo:

(13a) "Tomar poca sal es lo mejor para la salud, dicen los especialistas, pero yo no lo creo, siempre he tomado mucha sal y estoy hecho un roble."

(13b) "Tomar poca sal es lo mejor para la salud, dicen los especialistas, pero yo no me fiaría, nos dicen cualquier cosa para manipularnos."

Como se observa en los anteriores ejemplos, la disociación con la información y/o la fuente (13 a-b) se puede producir fácilmente si el hablante añade una marca explícita de desacuerdo, tal como lo explica AnderBois (2014: 243): "Crucial to establishing such context in many of the attested examples is the presence of other linguistic elements which serve to make the disconnect between the speaker's perspective and the reporter's publicly clear". El uso de dichos elementos permite atenuar el riesgo que conlleva el cambio de perspectiva (intencionado) que se produce. Este riesgo consiste en que el oyente podría malinterpretar lo dicho por el hablante y entender que la opinión reportada es también la suya o que la suscribe ${ }^{6}$ (Harris y Potts, 2009, citado en AnderBois, 2014: 243). Asimismo, la presencia de otro tipo de elementos evaluativos (de polaridad positiva) podría producir un efecto opuesto, como en (13c):

(13c) "Tomar poca sal es lo mejor para la salud, dicen los especialistas, y tienen toda la razón del mundo."

En cuanto al ejemplo (14), con o eso dicen no es necesaria ninguna marca adicional para que se vea claramente la disociación. Sin embargo, como hemos podido comprobar en el análisis del corpus, en un $25 \%$ de los casos (49 ejemplos), sí que se observa alguna marca explícita de desacuerdo, duda o negación que refuerza esta interpretación. Ocurren siempre con usos que hemos clasificado como reportativos o de rumor, es decir, no se dan en contextos de folclore.

(15) "Mientras no se presenten datos fiables, contrastados e independientes, decir que funciona porque la tia Paqui mejoró (o eso dicen) despues de tomarlo, es faltar a la verdad" (CDE/WebDialects, lacienciaysusdemonios.com)

\footnotetext{
6 "As Harris \& Potts (2009) discuss for appositives and expressives, perspective shift of this sort is a risky communicative strategy since the addressee may fail to understand the speaker's intended shift. The use of evaluative and other perspectival language in the denials mitigates this risk". (AnderBois, 2014: 243)
} 
En el ejemplo (15), se pone en duda la eficacia de un tratamiento señalando que faltan datos fiables para demostrarlo además de la opinión de algunas personas que dicen que gracias al dicho tratamiento "la tía Paqui mejoró”.

(16) "La cosa es que nuestra sorpresa es enorme cuando se nos muestra a Aegon vivito y coleando en Danza de Dragones. El hijo de Rhaegar nunca murió (o eso dicen, porque yo ya no sé de quién fiarme) y, según su padre, sería el Príncipe Prometido y un válido jinete de dragón" (CDE/WebDialects, eneltronodehierro.com)

En (16), se discuten algunos hechos relacionados con la trama de una serie fantástica en la que, por lo visto, hay algunas incógnitas. Una de ellas es la historia del personaje que se identifica como "hijo de Rhaegar" y que, según algunas fuentes "nunca murió". El hablante señala que no se fía ni de la información sobre la inmortalidad del personaje ni de su fuente. Aunque el uso de o eso dicen ya lo sugiere, esta lectura se ve reforzada por medio de la cláusula "no ya no sé de quién fiarme".

Parece evidente, pues, que o eso dicen favorece la interpretación de la disociación del hablante con lo dicho, especialmente en el caso de los usos reportativos. Sin embargo, como hemos señalado, no se observan ejemplos de esta "disociación reforzada" en el caso de folclore. Por el contrario, cuando la pieza de información reportada procede de la sabiduría general, el hablante a menudo se muestra partidario de la misma (17 y 18) y la utiliza para fortalecer su linea de argumentación, pues es un argumento que parece fiable desde el punto de vista del oyente, ya que proviene de una fuente que es compartida por ambos (folclore, sabiduría general).

(17) "De una boda sale otra boda, o eso dicen, y así se cumple en el final de' Parenthood'" (CDE/WebDialects, blogs.formulatv.com)

(18) "Ciertamente sagunti, rectificar es de sabios, o eso dicen" (CDE/WebDialects, blogs.lasprovincias.es)

En los usos de o eso dicen con evidencias del tipo folclore no parece haber trazas adicionales de disociación, lo cual sugiere que el hablante no reconoce como muy arriesgado que se le atribuya una parte del compromiso o de la responsabilidad con lo dicho. Creemos que la razón para ello hay que buscarla en la perspectiva evocada por una evidencia del tipo folclore. Con las evidencias reportadas o citativas se trata de una evidencia mediada o procedente de otros, lo cual explica porque la perspectiva del hablante puede estar diametralmente opuesta (esta es la base de la excepcionalidad reportativa). Sin embargo, en el caso de folclore o conocimientos compartidos, si bien la evidencia no se basa en la experiencia directa sensorial del hablante, 
no obstante, se origina en la tradición oral o en el acervo común de las personas a las que el propio hablante también pertenece. El carácter universal y accesible de la fuente lleva a que esta se deba considerar como compartida entre los miembros de la misma comunidad, incluido el hablante. No es, por tanto, una perspectiva simplemente del otro, sino que lo es del otro, de todos y también, en parte, del propio hablante.

Estas consideraciones nos llevan a concluir que la naturaleza de folclore difiere de la evidencialidad reportativa. Esto idea va en línea de las observaciones de Bermúdez (2005) sobre el tipo de acceso prototípico en cada caso, y de las reservas de Lazard (2001) a la hora de considerar el folclore como un subtipo de evidencialidad reportativa.

\section{Conclusiones}

En este artículo hemos analizado el comportamiento discursivo de la construcción o eso dicen con respecto a los diferentes valores de significado evidencial. Según los datos del análisis, esta construcción se utiliza con el valor reportativo, es decir, señala que la información proviene de fuentes externas del hablante. No obstante, cabe distinguir algunos matices en este significado, relacionados con el patrón de inserción de la construcción, su fijación, el carácter de la fuente a la que se alude y el tipo de ámbito sobre el que actúa. Teniendo en cuenta estos factores, se descubre que o eso dicen puede introducir el significado de evidencialidad reportativa, de rumor y también de conocimientos compartidos o de folclore. Se utiliza, asimismo, para señalar la disociación del hablante hacia la información reportada y/o su fuente, aunque este uso parece ligado más a los usos reportativos que a los de folclore. Según se ha propuesto, esto se explica porque o eso dicen con el valor de folclore parece evocar, de algún modo, también la perspectiva del propio hablante quien se considera parte de una comunidad de hablantes que comparten los mismos dichos y las mismas creencias. Asimismo, se comprueba que la evidencialidad del tipo reportativo y la de folclore aportan valores discursivos distintos.

\section{REFERENCIAS}

AIKHENVALD, A. Y. (2004): Evidentiality, Oxford: Oxford University Press.

AIKHENVALD, A. Y. (2007): "Information source and evidentiality: what can we conclude?", Rivista Di Linguistica, 19(1), pp. 209-227.

AIKHENVALD, A. Y. (ed.) (2018): The Oxford Handbook of Evidentiality, Oxford: Oxford University Press.
ALBELDA, M. (2015): "Introduction: Evidentiality in non-evidential languages: Are there evidentials in Spanish?", Journal of Pragmatics, 85, pp. 135-137.

ANDERBOIS, S. (2014): "On the exceptional status of reportative evidentials", Proceedings of SALT, 24, pp. 234-254.

BERMÚDEZ, F. (2005): Evidencialidad. La codificación lingüística 
del punto de vista, tesis doctoral, Stockholm: Stockholms Universitet.

CHAFE, W. L. (1986): "Evidentiality in English Conversation and Academic Writing", Chafe, W. L. y Nichols, J. (eds.): Evidentiality: The Linguistic Coding of Epistemology, Norwood, New Jersey: Ablex Publishing Corporation, pp. 261-272.

CORNILLIE, B. (2016): "Las lecturas evidenciales de los verbos (semi)auxiliares en español", González Ruiz, R. et al. (eds.): La evidencialidad en español: teoria y descripción, Frankfurt am Main: Iberoamericana Vervuert, pp. 227-250.

DE LA MORA, J. \& MALDONADO, R. (2015): "Dizque: Epistemics blurring evidentials in Mexican Spanish", Journal of Pragmatics, 85, pp. 168-180.

ESCANDELL VIDAL, M. V. (2010): "Futuro y evidencialidad", Anuario de Lingüística Hispánica, 25, pp. 9-34.

FALLER, M. (2002): Semantics and Pragmatics of evidentials in Cuzco Quechua, tesis doctoral.

GARCÍA NEGRONI, M. M. (2016): "Polifonía, evidencialidad citativa y tiempos verbales. Acerca de los usos citativos del futuro morfológico y del futuro perifrástico", González Ruiz, R. et al. (eds.): La evidencialidad en español: teoria y descripción, Frankfurt am Main: Iberoamericana Vervuert, pp. 279-301.

GONZÁLEZ VÂZQUEZ, M. (2016): "La naturaleza y función de la evidencialidad en español”, González Ruiz, R. et al. (eds.): La evidencialidad en español: teoria y descripción, Frankfurt am Main: Iberoamericana Vervuert, pp. 49-74.

GRAS, P. (2016): "Entre la codificación y la inferencia. Los valores cita- tivos de que inicial átono en español", González Ruiz, R. et al. (eds.): La evidencialidad en español: teoría y descripción, Frankfurt am Main: Iberoamericana Vervuert, pp. 201-223.

HASSLER, G. (2010): "Epistemic modality and evidentiality and their determination on a deictic basis: the case of Romance languages", Diewald, G. y Smirnova, E. (eds.): Linguistic Realization of Evidentiality in European Languages, Berlín-Nueva York: de Gruyter Mouton, pp. 223-248.

HARRIS, J. A. \& POTTS, C. (2009): "Perspective-shifting with appositives and expressives" Linguistics \& Philosophy, 32(6), pp. 523-552.

LAMPERT G. \& LAMPERT, M. (2010): "Where does evidentiality reside? Notes on (alleged) limiting cases: seem and be like", STUF - Language Typology and Universals Sprachtypologie und Universalienforschung, 63 (4), pp. 308-321.

LAZARD, G. (2001): "On the grammaticalization of evidentiality", Journal of Pragmatics, 33, pp. 359-367.

MiGLiO, V. G. (2010): "Online databases and language change: The Case of Spanish dizque", Gries, S.Th. et al. (eds.): Corpus Linguistics Applications: Current Studies, New Directions (Language and Computer Series), Amsterdam: Rodopi pp. 728.

PLUNGIAN, V. A. (2001): "The place of evidentiality within the universal grammatical space", Journal of Pragmatics, 33, pp. 349-357.

PLUNGIAN, V. (2010): "Types of verbal evidentiality marking: an overview", Diewald, G. y Smirnova, E. (eds.): Linguistic Ralization of 
Evidentiality in European Languages, Berlín-Nueva York: de Gruyter Mouton, pp. 15-58.

REYES, G. (1994): Los procedimientos de cita: citas encubiertas y ecos. Madrid: Arco/Libros.

RODRÍGUEZ ROSIQUE, S. (2015):

"Distance, evidentiality and counter-argumentation: Concessive future in Spanish", Journal of Pragmatics 85, pp. 181-199.

WILLET, T. (1988): "A Cross-Linguistic Survey of the Grammaticalization of Evidentiality", Studies in Language, 12(1), pp. 51-97. 